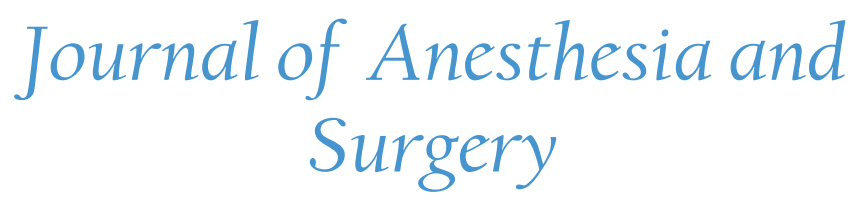

\title{
Tension Pneumothorax during Liver and Kidney Transplantation in a Patient with Adult Polycystic Kidney Disease
}

\author{
Fouad G. Souki, MD*, Georges Nasrallah, MD \\ Department of Anesthesiology, University of Miami, Jackson Health System, Miami, Florida, USA
}

"Corresponding author: Fouad G. Souki, University of Miami/Jackson Health System, 1611 NW $12^{\text {th }}$ Ave, DTC 318, Miami, FL 33136, Tel: 305-585-7435, Fax: 305-585-7477, E-mail: Fsouki@miami.edu

\begin{abstract}
Administering anesthesia to patients undergoing liver transplantation often yields intraoperative hemodynamic instability. During this type of major surgery, many factors may contribute to hemodynamic collapse, including myocardial infarction, pulmonary embolus, bleeding, and preload reduction owing to inferior vena cava clamping. Pneumothorax, a potentially dangerous condition, can arise unexpectedly during anesthesia. Diagnosis is based on exclusion, as the initial vital sign changes, including cardiorespiratory decompensation, are non-specific. Here, we describe the case of a patient with adult polycystic kidney disease who developed intraoperative cardiovascular collapse and hypoxemia owing to tension pneumothorax during liver and kidney transplantation. We highlight the etiology of intraoperative tension pneumothorax, the sequence of hemodynamic changes, and the importance of early diagnosis and treatment.
\end{abstract}

Keywords: Adult polycystic kidney disease; Tension pneumothorax; Liver transplantation; Kidney transplantation; Anesthesia
Received date: April 13, 2017

Accepted date: May 10, 2017

Published date: May 13, 2017

Citation: Souki, F.G., Nasrallah, G. Tension Pneumothorax during Liver and Kidney Transplantation in a Patient with Adult Polycystic Kidney Disease. (2017) J Anesth Surg 4(1): 47- 50.

DOI: $10.15436 / 2377-1364.17 .071$

\section{Introduction}

The recognition and management of intraoperative pneumothorax is critical for avoiding adverse events and unfavorable outcomes. Since pneumothorax can deteriorate into tension pneumothorax, it is important for clinicians to maintain a high index of suspicion. The causes of this life-threatening condition may be related to anesthesia, surgery, or the patient's preoperative predisposition. Though rare, pneumothorax can reportedly also occur following the rupture of pulmonary cysts in patients with adult polycystic kidney disease (APKD) ${ }^{[1,2]}$. Here, we present the case of a patient with APKD who developed intraoperative cardiovascular collapse and hypoxemia owing to tension pneumothorax during liver and kidney transplantation. We highlight possible etiologies of tension pneumothorax, sequence of hemodynamic changes, diagnostic challenge during liver transplantation, and importance of early recognition and treatment.

\section{Case Report}

A 56-year-old man with a history of APKD-related end-stage liver and renal disease presented for liver and kidney transplantation. The patient's weight and height were $65 \mathrm{~kg}$ and $177 \mathrm{~cm}$, respectively. Preoperative echocardiograms and dobutamine stress tests revealed normal sinus rhythms and cardiac function. The patient had no respiratory complaints, and a chest $\mathrm{X}$-ray showed clear lung fields. The patient had been placed on hemodialysis 2 years prior. Based on laboratory data obtained on the day of surgery, his Model for End-Stage Liver Disease score was 20 .

After attaching standard monitors, general anesthesia was induced intravenously with propofol $(150 \mathrm{mg})$, fentanyl $(100 \mu \mathrm{g})$, and rocuronium $(80 \mathrm{mg})$. An endotracheal tube was inserted uneventfully, and clear and symmetrical breath sounds were heard bilaterally upon auscultation of the lungs. Anesthesia was maintained with sevoflurane and $40 \%$ oxygen. Two large 
bore catheters (12 Fr and $9 \mathrm{Fr}$ ) were inserted, without difficulty, in the right internal jugular vein using ultrasound guidance. The hemodynamic and ventilation parameters were all within the normal limits (peak inspiratory pressure $\left[\mathrm{P}_{\mathrm{IP}}\right], 16 \mathrm{mmHg}$; tidal volume, $500 \mathrm{~mL}$ ). After the initial surgical incision, at $\sim 90 \mathrm{~min}$ after anesthesia induction, a significant decrease in blood pressure (BP) was noted $(53 / 30 \mathrm{mmHg}$ ) (Figure 1A). This hemodynamic change was attributed to surgical bleeding and inferior vena cava compression that commonly occurred during dissection and manipulation of the liver. A bolus of intravenous crystalloid $(500 \mathrm{~mL})$ along with multiple boluses of phenylephrine (total, $500 \mu \mathrm{g}$ ) improved BP (145/75 $\mathrm{mmHg})$. The surgeon was informed of the situation and asked to avoid major manipulation. Several minutes later, a second episode of severe hypotension occurred (systolic BP, $69 / 40 \mathrm{mmHg}$ ) that did not respond to administration of phenylephrine $(500 \mu \mathrm{g})$ and intravenous fluids $(500 \mathrm{~mL})$ (Figure 1B). Intravenous epinephrine $(100 \mu \mathrm{g})$ transiently raised the BP. Concurrently, the central venous pressure (CVP) increased from 5 to $14 \mathrm{mmHg}$, the $\mathrm{P}_{\mathrm{IP}}$ increased from 17 to $24 \mathrm{mmHg}$, and the oxyhemoglobin saturation dropped to $79 \%$ with $\mathrm{FiO}_{2}$ of $40 \%$. We switched to manual ventilation of the lungs and $100 \% \mathrm{FiO}_{2}$; the patient's oxyhemoglobin saturation recovered to $96 \%$. Upon lung auscultation, no breath sounds were heard on the right side. Meanwhile, the surgeon performing the liver dissection noticed that the right hemithorax was markedly distended in the surgical field. A right chest tube was inserted with immediate release of air through the drainage system. The air leak continued throughout surgery with a noticeable decrease in expiratory tidal volume. Nevertheless, the hemodynamic instability resolved immediately after chest tube insertion.

The operation resumed, and both the liver and kidney were successfully transplanted. During the operation, the patient received $6750 \mathrm{~mL}$ of packed red blood cells, $4500 \mathrm{~mL}$ of fresh frozen plasma, $500 \mathrm{~mL}$ of platelets, and $200 \mathrm{~mL}$ of cryoprecipitate. He was extubated $36 \mathrm{~h}$ later and discharged home 12 days after surgery.

\section{Discussion}

Tension pneumothorax has been reported in different types of surgeries under general anesthesia ${ }^{[3-6]}$. Mvarez reported intraoperative tension pneumothorax during cranioplasty in a patient with a remote history of trauma, while Reekie reported it during breast reconstruction surgery ${ }^{[3,4]}$. Other reports described tension pneumothorax during lung resection, and after using airway exchange catheter ${ }^{[5,6]}$. In many situations, the diagnosis of tension pneumothorax was not immediately clear and followed a preexisting condition or procedure. During liver transplantation, significant hemodynamic (bleeding, low systemic vascular resistance, surgical manipulation) and respiratory (hepatopulmonary syndrome, pleural effusion, atelectasis) changes are frequently present. This makes the early recognition of tension pneumothorax difficult. In our case, as in most liver transplant surgeries, the first episode of severe hemodynamic instability (Figure 1A) was most likely due to surgical manipulation since there was no change in $\mathrm{P}_{\mathrm{IP}}$, CVP or oxygen saturation. However, the second episode of severe hemodynamic instability (Figure 1B) had more of the classic signs of tension pneumothorax (decrease BP, increase CVP, increase $P_{I \text { P }}$, decrease oxygen saturation, absence of breaths sounds). What made the diagnosis definitive was the ipsilateral diaphragmatic bulging noted by the surgeon. From the anesthesiologist's standpoint, there was little to suspect a pneumothorax with an uncomplicated central line insertion and the rare incidence of APKD cysts in the lung. However, management was prompt which avoided further deleterious effects.

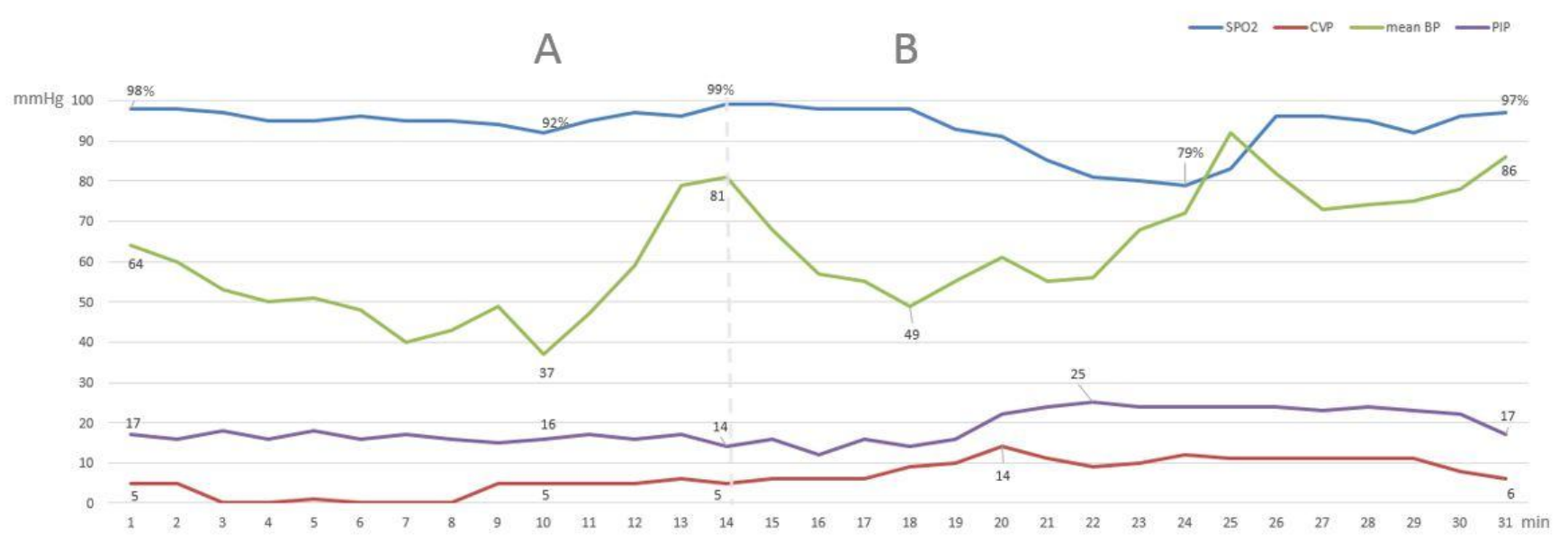

Figure 1: (A) Hemodynamic changes (mean $\mathrm{BP}, \mathrm{SPO}_{2}$ ) due to surgical manipulation with resolution after phenylephrine and fluid bolus. (B) Hemodynamic changes (mean BP, CVP, PIP, $\mathrm{SPO}_{2}$ ) due to tension pneumothorax with improvement after administration of epinephrine, $100 \% \mathrm{FiO}_{2}$ and chest tube insertion.

Pneumothorax is classified as primary or secondary based on the absence/presence of underlying respiratory pathology ${ }^{[7]}$. The incidence of pneumothorax during general anesthesia is low, reportedly accounting for $<3 \%$ of respiratory-related adverse outcomes ${ }^{[8]}$. The different causes of pneumothorax include regional nerve blocks ( $40 \%$ of reported cases), airway instrumentation $(19 \%)$, barotrauma $(16 \%)$, and central venous line placement $(7 \%)^{[9]}$. Patients with chronic obstructive pulmonary disease also have an increased risk of developing pneumothorax ${ }^{[10]}$. Likewise, the rupturing of preexisting emphysematous bullae can result in pneu$\operatorname{mothorax}^{[11]}$. 
Tension pneumothorax, usually defined as hemodynamic compromise in a patient with an expanding intrapleural air mass, is a rare, yet potentially disastrous, clinical diagno$\operatorname{sis}^{[10]}$. The initial clinical finding of tension pneumothorax during surgery is significant hypoxemia or hypotension. Later, cardiovascular collapse can develop ${ }^{[12]}$.

Pneumothorax can develop within a few minutes after lung injury, especially in patients on positive-pressure ventilation. In such cases, the underlying lung parenchymal injury can act as a one-way valve that allows air to enter the intrapleural space during inspiration but prohibits air from exiting during expiration. The resultant expanding air mass exerts pressure that collapses the ipsilateral lung and shifts the mediastinum toward the contralateral side. This shift is associated with compression of the vena cava and right heart structures, which causes decreased diastolic filling and cardiac output ${ }^{[13]}$. Shunting of the blood to underventilated lung areas with subsequent ventilation-to-perfusion mismatch also occurs. The result is hypoxemia, acidosis, and shock ${ }^{[14]}$. Compensatory mechanisms, including an elevated heart rate and increased respiratory excursion, may initially help preserve the hemodynamics in spontaneously breathing patients. Interestingly, the mechanical compressive effects of tension pneumothorax seem to occur earlier in subjects on positive-pressure ventilation. However, at what stage compressive forces become significant enough to cause tension pneumothorax remains unclear. Substantial hemodynamic compromise, or "tension physiology," is a late finding and indicates a preterminal event requiring immediate intervention ${ }^{[11]}$.

Studies of increasing intrapleural pressure in ventilated animals have demonstrated that progressive hypoxia is the earliest sign, along with an immediate rise in central venous pressure ${ }^{[15]}$. Another study that induced progressive tension pneumothorax in animals that were breathing room air showed that the $\mathrm{O}_{2}$ saturation dipped below $50 \%$ prior to cardiovascular collapse $^{[16]}$. Moreover, hypotension and tachycardia developed when the pneumothorax involved $57 \%$ of the total lung capacity, and sudden cardiovascular collapse occurred when the pneumothorax involved $>90 \%$ of the total lung capacity. Concurrent equalization of the mean intrapleural pressure with the CVP occurred at the point of collapse, and death occurred soon after ${ }^{[16]}$.

Hypoxemia may be less severe in mechanically ventilated patients who are receiving supplemental oxygen at the time of cardiovascular collapse. In our case, the early stage of pneumothorax development was marked by a decrease in BP followed by a decrease in oxygen saturation. This may be explained by the fact that the patient had been receiving $40 \%$ oxygen while mechanically ventilated. Furthermore, the administration of $100 \% \mathrm{FiO}_{2}$ increased the oxygen saturation back to $96 \%$ even before chest tube insertion.

Several mechanisms may explain the etiology of pneumothorax and subsequent tension pneumothorax during mechanical ventilation in our patient. One such explanation is inadvertent pleural puncture during central venous line insertion through the right internal jugular vein. Iatrogenic pneumothorax during central line placement has an incidence of $1-6 . \%^{[17]}$, with higher incidences related to subclavian vein insertion, emergencies, large catheter sizes, catheterization attempts, the physician's level of experience, and the patient's catheterization history ${ }^{[18]}$. In our case, an experienced anesthesiologist performed the two large central lines insertion using ultrasound guidance, yet without any suspicion of lung injury. However, even an uncomplicated central line insertion can lead to a pneumothorax.

Another possible explanation is that since our patient had APKD, pulmonary cysts may have ruptured intraoperatively owing to mechanical ventilation, leading to tension pneumothorax. The incidence of pulmonary cysts is rare in $\mathrm{APKD}^{[19]}$ and such cases are seldom described ${ }^{[2,20]}$. To date, only two reports have been published regarding recurrent pneumothorax associated with APKD-related pulmonary cysts. In one report, computed tomography images of a patient with persistent pneumothorax revealed cysts in the lungs, liver, and kidneys ${ }^{[2]}$. In the other report, a patient with a history of APKD presented with repeated episodes of spontaneous pneumothorax ${ }^{[1]}$. Unfortunately, the presence of cysts in the lungs could not be confirmed in our patient because no postoperative chest computed tomography was performed.

Knowing the pathophysiology and recognizing the hemodynamic and respiratory changes that occur with tension pneumothorax are critical for making an accurate diagnosis. One should keep in mind that even a small pneumothorax can become tension pneumothorax with positive-pressure ventilation during general anesthesia ${ }^{[21]}$. Once tension pneumothorax is diagnosed, high-flow oxygen therapy should be started immediately to maximize oxygenation and to provide an oxygen reservoir for further resuscitation. Concurrently, emergency needle decompression followed by tube thoracotomy should be performed ${ }^{[22]}$. In our patient, after $15 \mathrm{~min}$ of hemodynamic instability and tension physiology, the insertion of a chest tube prevented cardiopulmonary arrest.

The incidence of death remains unclear but tension pneumothorax appears to be more serious in ventilated patients, with one study showing a $91 \%$ mortality rate ${ }^{[23]}$. Death among mechanically ventilated patients was reportedly $\sim 38$ times higher in those who developed tension pneumothorax than it was in those who did not ${ }^{[23]}$. Delayed or missed diagnoses can result in poor outcomes ${ }^{[24]}$.

\section{Conclusion}

During liver transplantation, various factors may lead to the development of hemodynamic collapse. Recognizing a patient's preoperative predisposition to develop pneumothorax (central line insertion) and understanding whether pneumothorax is a common or rare manifestation of a disease (APKD) is important. Pneumothorax may become tension pneumothorax with the application of positive-pressure ventilation. Limited access to the chest during anesthesia may hinder diagnosis. Significant hypoxemia occurs early and precedes hypotension owing to occlusive mechanical compression. However, the occurrence of hypoxemia may be delayed or less severe in patients receiving supplemental oxygen. Prompt diagnosis and communication with the surgical team along with hemodynamic support, needle aspiration, and insertion of a chest drain are essential for a favorable outcome.

Acknowledgements: We would like to thank Editage (www.editage.com ) for the English language editing.

Conflicts of interest: The authors declare no conflict of interest. 


\section{References}

1. DiNuoscio, D., Botros, N. Multiple Recurrent Pneumothoraces In A Patient With Polycystic Kidney Disease: A Case Report And Literature Review. (2014) Am J Respir Crit Care Med 189: A6107.

Others

2. Shanmuganathan, A., Rao, U.K., Chandrasekharan, A., et al. Recurrent Pneumothorax Associated with Polycystic Kidney. (2008) Indian J Chest Dis Allied Sci 50(2): 233-235.

Others

3. Mavarez-Martinez, A., Soghomonyan, S., Sandhu, G., et al. Intraoperative Tension Pneumothorax in a Patient with Remote Trauma and Previous Tracheostomy. (2016) J Investig Med High Impact Case Rep 4(1): 2324709616636397.

Pubmed | Crossref

4. Reekie, T., McGill, D., Marshall, E. Diagnosing intraoperative pneumothorax in patients undergoing autologous breast reconstruction: a useful clinical sign. (2014) Case Rep Surg 2014: 308485.

Pubmed | Crossref | Others

5. Finlayson, G.N., Chiang, A.B., Brodsky, J.B., et al. Intraoperative contralateral tension pneumothorax during pneumonectomy. (2008) Anesth Analg 106(1): 58-60.

Pubmed | Crossref | Others

6. Hulst, A.H., Avis, H.J., Hollmann, M.W., et al. Massive Subcutaneous Emphysema and Bilateral Tension Pneumothoraces After Supplemental Oxygen Delivery via an Airway Exchange Catheter: A Case Report. (2017) A A Case Rep 8(2): 26-28.

Pubmed | Crossref | Others

7. MacDuff, A., Arnold, A., Harvey, J. Management of spontaneous pneumothorax: British Thoracic Society Pleural Disease Guideline 2010. (2010) Thorax 65(Suppl 2): ii18-31.

Pubmed | Crossref | Others

8. Cheney, F.W., Posner, K.L., Caplan, R.A. Adverse respiratory events infrequently leading to malpractice suits. A closed claims analysis. (1991) Anesthesiology 75(6): 932-939.

Pubmed | Others

9. Ibrahim, A.E., Stanwood, P.L., Freund, P.R. Pneumothorax and systemic air embolism during positive-pressure ventilation. (1999) Anesthesiology 90(5): 1479-1481.

Pubmed | Others

10. Barton, E.D. Tension pneumothorax. (1999) Curr Opin Pulm Med 5(4): 269-74.

Pubmed

11. Zollinger, A., Zaugg, M., Weder, W., et al. Video-assisted thoracoscopic volume reduction surgery in patients with diffuse pulmonary emphysema: gas exchange and anesthesiological management. (1997) Anesth Analg 84(4): 845-851.

Pubmed | Others
12. Fossard, J.P., Samet, A., Meistelman, C., et al. Life-threatening pneumothorax of the ventilated lung during thoracoscopic pleurectomy. (2001) Can J Anaesth 48(5): 493-496.

Pubmed | Crossref | Others

13. Simpson, G., Vincent, S., Ferns, J. Spontaneous tension pneumothorax: what is it and does it exist? (2012) Intern Med J 42(10): 1157-1160. Pubmed | Crossref

14. Steier, M., Ching, N., Roberts, E.B., et al. Pneumothorax complicating continuous ventilatory support. (1974) J Thorac Cardiovasc Surg 67(1): 17-23

Pubmed | Others

15. Carvalho, P., Hilderbrandt, J., Charan, N.B. Changes in bronchial and pulmonary arterial blood flow with progressive tension pneumothorax. (1985) J Appl Physiol 81(4): 1664-1669.

Pubmed | Others

16. Barton, E.D., Rhee, P., Hutton, K.C., et al. The pathophysiology of tension pneumothorax in ventilated swine. (1997) J Emerg Med 15(2): 147-153.

Pubmed | Crossref | Others

17. Kusminsky, R.E. Complications of central venous catheterization. (2007) J Am Coll Surg 204(4): 681-696.

Crossref

18. Tsotsolis, N., Tsirgogianni, K., Kioumis, I., et al. Pneumothorax as a complication of central venous catheter insertion. (2015) Ann Transl Med 3(3): 40.

Others

19. Moua, T., Zand, L., Hartman, R.P., et al. Radiologic and clinical bronchiectasis associated with autosomal dominant polycystic kidney disease. (2014) PLoS One 9(4): e93674.

Crossref

20. Li, H.Y., Cosgrove, G.P., Swigris, J.J. Dyspnea in a 43-year-old woman with polycystic kidney disease. (2009) Chest 135(1): 238-243. Pubmed | Crossref

21. Bacon, A.K., Paix, A.D., Williamson, J.A., et al. Crisis management during anaesthesia: pneumothorax. (2005) Qual Saf Health Care 14(3): e18.

Pubmed | Crossref | Others

22. Barton, E.D., Epperson, M., Hoyt, D.B., et al. Prehospital needle aspiration and tube thoracostomy in trauma victims: a six-year experience with aeromedical crews. (1995) J Emerg Med 13(2): 155-163.

Crossref

23. Chen, K.Y., Jerng, J.S., Liao, W.Y., et al. Pneumothorax in the ICU: patient outcomes and prognostic factors. (2002) Chest 122(2): 678-683. Pubmed | Crossref

24. Leigh-Smith, S., Harris, T. Tension pneumothorax--time for a rethink? (2005) Emerg Med J 22(1): 8-16.

Pubmed | Crossref
Ommega Online Publishers

Journal Title: Journal of Anesthesia and Surgery (JAS)

Journal Short Name: J Anesth Surg
Journal ISSN: 2377-1364

E-mail: anestheisa@ommegaonline.com Website: www.ommegaonline.org 